\title{
Quartic fermion self-interactions in two-dimensional gauge theories
}

\author{
A. S. Dutra \\ Departamento de Física e Química, Universidade Estadual Paulista, Campus de Guaratinguetá, Caixa Postal 205, \\ 12500-000 Guaratinguetá, São Paulo, Brazil \\ C. P. Natividade \\ Instituto de Física, Universidade Federal Fluminense, Avenida Litoranea, S/N, CEP 24210-340, Niteroi, Rio de Janeiro, Brazil \\ H. Boschi-Filho* \\ Instituto de Física, Universidade Federal do Rio de Janeiro, Cidade Universitária, Ilha do Fundão, Caixa Postal 68528, \\ 21945-970 Rio de Janeiro, Brazil \\ R. L. P. G. Amaral and L. V. Belvedere \\ Instituto de Física, Universidade Federal Fluminense, Avenida Litoranea, S/N, CEP 24210-340, Niteroi, Rio de Janeiro, Brazil
}

(Received 1 November 1996)

\begin{abstract}
In this work we discuss the effect of quartic fermion self-interacting terms on the dynamically generated photon masses in $1+1$ dimensions, for vector, chiral, and non-Abelian couplings. In the vector and chiral cases we find exactly the dynamically generated mass modified by the quartic term while in the non-Abelian case we find the dynamically generated mass associated with its Abelian part. We show that in the three cases there is a kind of duality between the gauge and quartic couplings. We perform functional as well as operator treatments allowing for the obtention of both fermion and vector field solutions. The structures of the Abelian models in terms of $\theta$ vacua are also addressed. [S0556-2821(97)02208-X]
\end{abstract}

PACS number(s): 11.10.Kk, 11.15.Tk

\section{INTRODUCTION}

Two-dimensional models in quantum field theory provide rich situations where many interesting physical properties, which are common to higher-dimensional models, can be studied as screening, confinement, $\theta$ vacua, etc. The main advantage of modeling in low dimensions is that, in general, integrability can be improved as one lowers the dimensions. One of the best known of these two-dimensional models is the (vector) Schwinger model [1] which was solved using the operator approach in the classical paper by Lowenstein and Swieca [2]. A functional solution to it, using the Fujikawa's method [3], was also given later [4]. The introduction of a consistent model with anomalously broken gauge symmetry, the chiral Schwinger model [5], renewed the interest for gauge theories in two dimensions [6]. The generalization to arbitrary left and right coupling in [7] was particularly studied using the interaction picture method for obtaining the bosonized Lagrangian in [8].

On the other hand, the study of purely fermionic interacting models has been also developed since the introduction of the Thirring model [9] that was completely bosonized in the classical paper by Klaiber [10]. Later on, there was a growing interest in discussing generalizations to non-Abelian fermionic models as in the one proposed by Gross and Neveu [11], which is exactly solvable in the large $N$ limit. The study of the chiral Gross-Neveu model was performed by adding

\footnotetext{
*Present address: Center for Theoretical Physics, Laboratory for Nuclear Science, Massachusetts Institute of Technology, Cambridge, Massachusetts.
}

the interaction with an Abelian gauge field [12] or with diagonal $\mathrm{SU}(N)$ gluon fields [13]. These embeddings shed some light on the dynamical mass generation mechanism of the Gross-Neveu model. In these cases the Gross-Neveu model is not completely solvable through the bosonization formalism and the U(1) scalar field that couples to the Abelian gauge field is a free one decoupled from the interacting infrafermions.

Another important development in two-dimensional field theory was gained with the string and superstring theories, since the manifold of these objects are two dimensional and the machinery for this specific dimension, as conformal invariance could be plainly used [14]. In particular, the interest for these theories was recently renewed by the discovery of duality in the supersymmetric formulation [15].

Our aim here is to study two-dimensional models where fermions couple to gauge fields and to themselves via a quartic interaction, mainly to see the effect of the fermion selfcoupling in the dynamically generated mass and also to discuss the physical properties of these models, which may differ from the original ones, without quartic interaction. Incidentally, the scalars that will be coupled to the gauge fields will be affected by the quartic interactions.

We first take, in Sec. II, the vector Schwinger model and add a $J^{\mu} J_{\mu}$ interaction. We solve it exactly by the functional method, using an identity, with the introduction of an auxiliary gauge field, which turns the self-interaction term into a modified gauge coupling. We find that the mass acquired by the vector field is modified by the original self-interaction coupling. Then, in Sec. III, we study an analogous extension for the chiral Schwinger model by introducing also a selfinteraction fermion term. Although there is no quartic fermi- 
onic term preserving chirality for free fermions, the ambiguity in the definition of the Jacobian of the functional measure makes it worthwhile to consider this model, for which we also find exact solutions. Next, in Sec. IV, we discuss the case of non-Abelian fermions coupled to non-Abelian vector fields. In contrast with the Abelian cases, this one is not exactly solvable; however, we can determine again the dynamically generated mass taking into account only the Abelian part of the gauge fields.

We find in these three situations a kind of duality between the electromagnetic $e$ and the quartic self-interaction $g$ couplings.

To get more confidence of these results, we also study the vector and chiral (Abelian) models in the operator formalism for which explicitly solutions are obtained, in Secs. V and VI, respectively. This treatment allows the obtention of the fermionic correlation functions and gives the spurious operators related to the $\theta$ vacua. We find that the physical properties of the models with quartic self-interaction are similar to the ones in the models in which they are not present. Specifically, in the vector case there are operators which violate the cluster property and so, for consistency, it is necessary to define a $\theta$ vacuum. However, the spurious operators which determine these physical properties depend on the self-interaction coupling and are not chirally invariant as usual. In the chiral case the $\theta$ vacuum is absent as in the case without self-interaction. Finally, in Sec. VII, we present our conclusions.

\section{THE VECTOR MODEL}

For the vector gauge model in two dimensions with massless fermions, we have that the Lagrangian density, in Euclidean space within the topological trivial sector, can be written as

$$
\begin{aligned}
\mathcal{L}= & \bar{\psi}(i \theta-e A) \psi-\frac{g^{2}}{2}\left(\bar{\psi} \gamma_{\mu} \psi\right)^{2}+\frac{1}{4}\left(F_{\mu \nu}\right)^{2}+J_{\mu} A_{\mu}+\bar{\theta} \psi \\
& +\bar{\psi} \theta
\end{aligned}
$$

where $F_{\mu \nu}=\partial_{\mu} A_{\nu}-\partial_{\nu} A_{\mu}$. Using the identity

$$
\begin{aligned}
\exp ( & \left.+\frac{g^{2}}{2} \int d^{2} x\left(\bar{\psi} \gamma_{\mu} \psi\right)^{2}\right) \\
& =\frac{1}{\mathcal{N}} \int \mathcal{D} B_{\mu} \exp \left[-\int d^{2} x\left(\frac{e^{2}}{2}\left(B_{\mu}\right)^{2}-e g \bar{\psi} \gamma_{\mu} B_{\mu} \psi\right)\right]
\end{aligned}
$$

it turns to be (in the Lorentz gauge)

$$
\begin{aligned}
\mathcal{L}= & \bar{\psi}[i \not b-e(A+g \mathbb{B})] \psi+\frac{e^{2}}{2} B_{\mu} B^{\mu}+\frac{1}{4} F_{\mu \nu} F^{\mu \nu} \\
& -\frac{1}{2 \alpha}\left(\partial_{\mu} A^{\mu}\right)^{2}+\text { source terms. }
\end{aligned}
$$

This model is a special case of the one analyzed in [16]. Now, doing the transformation $A_{\mu}=\bar{A}_{\mu}-\bar{B}_{\mu}$, where $\bar{B}_{\mu}=g B_{\mu}$, the Lagrangian density becomes

$$
\begin{aligned}
\overline{\mathcal{L}}= & \bar{\psi}(i \not \partial+e \bar{A}) \psi+\frac{e^{2}}{2 g^{2}} \bar{B}_{\mu} \bar{B}^{\mu}+\frac{1}{4} \bar{F}_{\mu \nu} \bar{F}^{\mu \nu}+\frac{1}{4} \bar{M}_{\mu \nu} \bar{M}^{\mu \nu} \\
& -\frac{1}{2} \bar{F}_{\mu \nu} \bar{M}^{\mu \nu}-\frac{1}{2 \alpha}\left(\partial_{\mu} \bar{A}^{\mu}\right)^{2}-\frac{1}{2 \alpha}\left(\partial_{\mu} \bar{B}^{\mu}\right)^{2}+\frac{1}{\alpha}\left(\partial_{\mu} \bar{A}^{\mu}\right) \\
& \times\left(\partial_{\nu} \bar{B}^{\nu}\right)+\text { source terms },
\end{aligned}
$$

where $\bar{M}_{\mu \nu}=\partial_{\mu} \bar{B}_{\nu}-\partial_{\nu} \bar{B}_{\mu}$. In order to decouple the fermions and the gauge fields $\bar{A}_{\mu}$ and $\bar{B}_{\mu}$, we decompose the last two into their longitudinal and transversal parts through

$$
e \bar{A}_{\mu}=\partial_{\mu} \eta_{A}+\epsilon_{\mu \nu} \partial^{\nu} \chi_{A}, \quad e \bar{B}_{\mu}=\partial_{\mu} \eta_{B}+\epsilon_{\mu \nu} \partial^{\nu} \chi_{B}
$$

and perform the transformation

$$
\begin{gathered}
\psi(x)=\exp \left(-i \eta_{A}+\gamma_{5} \chi_{A}\right) \psi^{\prime}(x), \\
\bar{\psi}(x)=\bar{\psi}^{\prime}(x) \exp \left(i \eta_{A}+\gamma_{5} \chi_{A}\right)
\end{gathered}
$$

The corresponding nontrivial Jacobian is given by

$$
J_{F}=\exp \left(+\frac{\lambda}{2 \pi} \int d^{2} x\left(\chi_{A} \square \chi_{A}\right)\right)
$$

where $\lambda$ is the arbitrary regularization parameter. In this work we will restrict ourselves to $\lambda=1$. So, we get

$$
\begin{aligned}
\mathcal{L}_{\mathrm{eff}}= & i \bar{\psi}^{\prime} \partial \psi^{\prime}+\frac{1}{2 e^{2}}\left[\left(\square \chi_{A}\right)^{2}+\left(\square \chi_{B}\right)^{2}\right]-\frac{1}{e^{2}} \square \chi_{A} \square \chi_{B} \\
& +\frac{1}{2 g^{2}}\left[\left(\partial_{\mu} \eta_{B}\right)^{2}+\left(\partial_{\mu} \chi_{B}\right)^{2}\right]+\frac{1}{2 \pi}\left(\partial_{\mu} \chi_{A}\right)^{2} \\
& -\frac{1}{2 \alpha e^{2}}\left[\left(\square \eta_{A}\right)^{2}+\left(\square \eta_{B}\right)^{2}\right]+\frac{1}{\alpha e^{2}} \square \eta_{A} \square \eta_{B} \\
& +\frac{1}{e} J_{\mu}\left[\partial^{\mu}\left(\eta_{A}-\eta_{B}\right)+\epsilon_{\mu \nu} \partial^{\nu}\left(\chi_{A}-\chi_{B}\right)\right] \\
& +\bar{\theta} e^{-i \eta_{A}+\gamma_{5} \chi_{A}} \psi^{\prime}+\bar{\psi}^{\prime} e^{i \eta_{A}+\gamma_{5} \chi_{A} \theta .}
\end{aligned}
$$

Doing the rescale

$$
\chi_{A} \rightarrow a \bar{\chi}_{A}, \quad \chi_{B} \rightarrow \bar{\chi}_{B}, \quad a=\sqrt{\frac{\pi}{g^{2}}}
$$

and the field transformations

$$
\begin{gathered}
\left(\begin{array}{c}
\bar{\chi}_{A} \\
\bar{\chi}_{B}
\end{array}\right)=\left(\begin{array}{cc}
\cos \phi & \sin \phi \\
-\sin \phi & \cos \phi
\end{array}\right)\left(\begin{array}{l}
\chi_{A}^{\prime} \\
\chi_{B}^{\prime}
\end{array}\right), \\
\left(\begin{array}{l}
\eta_{A} \\
\eta_{B}
\end{array}\right)=\left(\begin{array}{ll}
1 & 1 \\
0 & 1
\end{array}\right)\left(\begin{array}{c}
\eta_{A}^{\prime} \\
\eta_{B}^{\prime}
\end{array}\right)
\end{gathered}
$$

the Lagrangian density (2.8) decouples when we choose $\tan \phi=1 / a$, so that we find 


$$
\begin{aligned}
\mathcal{L}_{\mathrm{eff}}^{\prime}= & i \bar{\psi}^{\prime} \phi \psi^{\prime}+\frac{1}{2 g^{2}}\left[\left(\partial_{\mu} \chi_{A}^{\prime}\right)^{2}+\left(\partial_{\mu} \chi_{B}^{\prime}\right)^{2}+\left(\partial_{\mu} \eta_{B}^{\prime}\right)^{2}\right] \\
& +\frac{a^{2}+1}{2 e^{2}}\left(\square \chi_{A}^{\prime}\right)^{2}-\frac{1}{2 \alpha e^{2}}\left(\square \eta_{A}^{\prime}\right)^{2} \\
& +\frac{1}{e} J^{\mu}\left(\partial_{\mu} \eta_{A}^{\prime}+\sqrt{a^{2}+1} \epsilon_{\mu \nu} \partial^{\nu} \chi_{A}^{\prime}\right) \\
& +\bar{\theta} \exp \left[-i\left(\eta_{A}^{\prime}+\eta_{B}^{\prime}\right)+\gamma_{5} a\left(\cos \phi \chi_{A}^{\prime}+\sin \phi \chi_{B}^{\prime}\right)\right] \psi^{\prime} \\
& +\bar{\psi}^{\prime} \exp \left[i\left(\eta_{A}^{\prime}+\eta_{B}^{\prime}\right)+\gamma_{5} a\left(\cos \phi \chi_{A}^{\prime}+\sin \phi \chi_{B}^{\prime}\right)\right] \theta .
\end{aligned}
$$

From this Lagrangian density we can infer the fundamental propagators of the theory, e.g., for the photon propagator we have

$$
\begin{aligned}
\mathcal{D}_{\mu \nu}(k) & =\left.\frac{\delta^{2} Z}{\delta J_{\mu} \delta J_{\nu}}\right|_{J=0} \\
& =-\frac{1}{e^{2}}\left[k_{\mu} k_{\nu} \mathcal{D}_{\eta_{A}^{\prime}}+\left(a^{2}+1\right) \epsilon_{\mu \sigma} \epsilon_{\nu \rho} k^{\sigma} k^{\rho} \mathcal{D}_{\chi_{A}^{\prime}}\right] .
\end{aligned}
$$

Using the two-dimensional identity $\epsilon_{\mu \sigma} \epsilon_{\nu \rho}=\delta_{\mu \nu} \delta_{\sigma \rho}-$ $\delta_{\mu \rho} \delta_{\sigma \nu}$, we obtain explicitly

$$
\mathcal{D}_{\mu \nu}(k)=-\frac{i}{k^{2}-m^{2}}\left[g_{\mu \nu}-\left(1-\frac{\alpha\left(k^{2}+m^{2}\right)}{k^{2}}\right) \frac{k_{\mu} k_{\nu}}{k^{2}}\right],
$$

where the dynamically generated mass is given by

$$
m^{2}=\frac{e^{2}}{\pi+g^{2}} .
$$

Here, we can see the effect of the fermion self-interaction on the dynamically generated mass $m$, keeping the gauge constant $e$ fixed and varying the quartic coupling constant $g$. So, for a vanishing $g$, one recovers the usual Schwinger mass $[1,2,4]$, and for the strong $g$ coupling one gets

$$
m^{2} \sim\left(\frac{e}{g}\right)^{2}, \quad(g \rightarrow \infty),
$$

showing a duality between the quartic and gauge coupling in the sense that the model with strong $g$ coupling has its vector field correlation functions equal to the ones of the Schwinger model with small electromagnetic coupling. This kind of duality also appears in the other models with quartic fermion self-coupling that we are going to describe, suggesting that this is a general behavior of these couplings rather than a particular model property.

\section{THE CHIRAL MODEL}

Let us now discuss the fermion self-interaction effect on the chiral massless two-dimensional electrodynamics. This discussion follows closely that of the previous section so we will skip many details. The Lagrangian density in this case can be written as

$$
\begin{aligned}
\mathcal{L}_{\text {chiral }}= & \bar{\psi}\left[i b-e\left(1-\gamma_{5}\right) A\right] \psi-\frac{g^{2}}{2}\left[\bar{\psi} \gamma_{\mu}\left(1+\gamma_{5}\right) \psi\right]^{2} \\
& +\frac{1}{4} F_{\mu \nu} F^{\mu \nu}+J_{\mu} A^{\mu}+\bar{\theta} \psi+\bar{\psi} \theta
\end{aligned}
$$

In order to rewrite the fermion self-interaction with the aid of an auxiliary field, we use the identity

$$
\begin{aligned}
\exp (+ & \left.\frac{g^{2}}{2} \int d^{2} x\left[\bar{\psi} \gamma_{\mu}\left(1+\gamma_{5}\right) \psi\right]^{2}\right) \\
= & \frac{1}{\mathcal{N}} \int \mathcal{D} B_{\mu} \exp \left[-\int d^{2} x\left(\frac{e^{2}}{2} B_{\mu} B^{\mu}-e g \bar{\psi} \gamma_{\mu}\right.\right. \\
& \left.\left.\times\left(1+\gamma_{5}\right) B^{\mu} \psi\right)\right]
\end{aligned}
$$

which after substitution in $\mathcal{L}_{\text {chiral }}$ and the transformation $A_{\mu}=\bar{A}_{\mu}-\bar{B}_{\mu}$, where $\bar{B}_{\mu}=g B_{\mu}$, it becomes

$$
\begin{aligned}
\overline{\mathcal{L}}_{\text {chiral }}= & \bar{\psi}\left[i \not b-e\left(1-\gamma_{5}\right) \bar{A}\right] \psi+\frac{e^{2}}{2 g^{2}} \bar{B}_{\mu} \bar{B}^{\mu}+\frac{1}{4} \bar{F}_{\mu \nu} \bar{F}^{\mu \nu} \\
& +\frac{1}{4} \bar{M}_{\mu \nu} \bar{M}^{\mu \nu}-\frac{1}{2} \bar{F}_{\mu \nu} \bar{M}^{\mu \nu}+\text { source terms. }
\end{aligned}
$$

Decomposing the boson fields in its longitudinal and transversal parts (2.5) and doing the transformation on Fermi fields

$$
\begin{gathered}
\psi(x)=\exp \left[-i \eta_{A}+\left(1-\gamma_{5}\right) \chi_{A}\right] \psi^{\prime}(x), \\
\bar{\psi}(x)=\bar{\psi}^{\prime}(x) \exp \left[i \eta_{A}+\left(1+\gamma_{5}\right) \chi_{A}\right],
\end{gathered}
$$

we find a nontrivial Jacobian given by

$$
\begin{aligned}
J_{\text {chiral }}= & \exp \left(-\frac{1}{2 \pi} \int d^{2} x\left[\alpha \eta_{A} \square \eta_{A}-\beta \chi_{A} \square \chi_{A}\right.\right. \\
& \left.\left.-(\alpha-\beta) \eta \square \chi_{A}\right]\right),
\end{aligned}
$$

where $\alpha$ and $\beta$ are the arbitrary regularization parameters in this case. Bringing back these results into $\mathcal{L}_{\text {chiral }}$, we find the effective Lagrangian density

$$
\begin{aligned}
\mathcal{L}_{\text {chiral }}^{\text {eff }}= & i \bar{\psi}^{\prime} \phi \psi^{\prime}+\frac{1}{2 e^{2}}\left[\left(\square \chi_{A}\right)^{2}+\left(\square \chi_{B}\right)^{2}\right]-\frac{1}{e^{2}} \square \chi_{A} \square \chi_{B} \\
& +\frac{1}{2 g^{2}}\left(\partial_{\mu} \chi_{B}\right)^{2}+\frac{1}{2 \pi}\left[\alpha \eta_{A} \square \eta_{A}-\beta \chi_{A} \square \chi_{A}\right. \\
& \left.-(\alpha-\beta) \eta_{A} \square \chi_{A}\right]+ \text { source terms. }
\end{aligned}
$$

Introducing the auxiliary field $\theta_{A}$ and doing the translation 


$$
\eta_{A} \rightarrow \theta_{A}+b \chi_{A},
$$

with the choice $b=(\alpha-\beta) / 2 \alpha$, in order to cancel the crossing term between $\theta_{A}$ and $\chi_{A}$, we find the Lagrangian density

$$
\begin{aligned}
\mathcal{L}_{\text {chiral }}^{\text {eff }}= & i \bar{\psi}^{\prime} \phi \psi^{\prime}+\frac{1}{2 e^{2}}\left[\left(\square \chi_{A}\right)^{2}+\left(\square \chi_{B}\right)^{2}\right]-\frac{1}{e^{2}} \square \chi_{A} \square \chi_{B} \\
& -\frac{1}{2 g^{2}} \chi_{B} \square \chi_{B}-\frac{1}{8 \pi} \frac{(\alpha+\beta)^{2}}{\alpha} \chi_{A} \square \chi_{A} \\
& -\frac{\alpha}{2 \pi} \theta_{A} \square \theta_{A}+\text { source terms. }
\end{aligned}
$$

Comparing this Lagrangian with the one given for the vector model (2.8) and noting that the dependence on $\theta_{A}$ can be absorbed in the normalization constant, we see that one can relate the vector model with this one with the substitution

$$
\frac{\lambda}{2 \pi} \rightarrow \frac{(\alpha+\beta)^{2}}{8 \pi \alpha} .
$$

Consequently, after a rotation analogous to Eq. (2.10), in order to decouple $\chi_{A}$ and $\chi_{B}$, we find that the dynamically generated mass for the chiral model is given by

$$
m^{2}=\frac{e^{2}(\alpha+\beta)^{2}}{4 \pi \alpha+g^{2}(\alpha+\beta)^{2}} .
$$

This mass is regularization dependent ( $\alpha$ and $\beta$ ) as happens in the original Jackiw-Rajaraman model [5]. We can reobtain their standard result letting $g \rightarrow 0$ (see also [18]). For the strong coupling limit $(g \rightarrow \infty)$, one gets the same kind of duality as that for the vector model (2.16), of the previous section.

\section{THE NON-ABELIAN CASE}

Let us now extend the previous discussion on quartic fermion self-interaction in the vector model to the nonAbelian case. So, we start here with the Lagrangian density

$$
\begin{aligned}
\mathcal{L}= & \bar{\psi}(i \not b-e A) \psi-\frac{g^{2}}{2 N}\left(\bar{\psi} \gamma_{\mu} \lambda^{a} \psi\right)^{2}+\frac{1}{4} \operatorname{tr} F_{\mu \nu} F^{\mu \nu}+J_{\mu} A^{\mu} \\
& +\bar{\theta} \psi+\bar{\psi} \theta,
\end{aligned}
$$

where $\lambda^{a},\left(a=0,1, \ldots, N^{2}-1\right)$ are the generators of the $\mathrm{U}(N)$ group, $F_{\mu \nu}=\partial_{\mu} A_{\nu}-\partial_{\nu} A_{\mu}+e\left[A_{\mu}, A_{\nu}\right], \psi$ and $\bar{\psi}$ transform under $\mathrm{U}(N)$ group, and we decompose the gauge field into its Abelian and non-Abelian parts $A_{\mu}=A_{\mu}^{0} \lambda^{0}+A_{\mu}^{a} t^{a}$, where $t^{a}=\lambda^{a},\left(a=1,2, \ldots, N^{2}-1\right) \quad$ generate the $\mathrm{SU}(N)$ group and $\lambda^{0}=I / \sqrt{N}\left(\operatorname{tr} t^{a} t^{b}=\delta^{a b}\right)$.

Contrary to the previous cases, the non-Abelian case is not exactly solvable, as happens for other non-Abelian twodimensional models [19]. However, one can factorize the Abelian part of the Lagrangian and take its contribution exactly, analogously to the approach of Furuya et al. for the chiral-invariant Gross-Neveu model [20]. As we will see, this contribution will imply a mass which also depends on the non-Abelian gauge group constant $N$.

Using the identity [20]

$$
\begin{aligned}
& \exp \left(\frac{g^{2}}{2 N} \int d^{2} x\left(\bar{\psi} \gamma_{\mu} \lambda^{a} \psi\right)^{2}\right) \\
& \quad=\frac{1}{\mathcal{N}} \int \mathcal{D} B_{\mu} \exp \left[-\int d^{2} x\left(\frac{1}{2} \operatorname{tr} B_{\mu} B^{\mu}-\frac{g}{\sqrt{N}} \bar{\psi} \gamma_{\mu} B^{\mu} \psi\right)\right],
\end{aligned}
$$

where $B_{\mu}=B_{\mu}^{0} I / \sqrt{N}+B_{\mu}^{a} t^{a}$ with the same symmetry group of the field $A_{\mu}$, so that

$$
\operatorname{tr} B_{\mu} B^{\mu}=B_{\mu}^{0} B^{0 \mu}+B_{\mu}^{a} B^{a \mu},
$$

the Lagrangian density becomes (apart from source terms)

$$
\mathcal{L}=\bar{\psi} \gamma^{\mu}\left(i \partial_{\mu}-e A_{\mu}-\frac{g}{\sqrt{N}} B_{\mu}\right) \psi+\frac{1}{2} \operatorname{tr} B_{\mu} B^{\mu}+\frac{1}{4} \operatorname{tr} F_{\mu \nu} F^{\mu \nu} .
$$

Now, doing the transformations $\bar{B}_{\mu}=(g / e \sqrt{N}) B_{\mu}$ and $A_{\mu}=\bar{A}_{\mu}-\bar{B}_{\mu}$, we have

$$
\begin{aligned}
\overline{\mathcal{L}}= & \bar{\psi} \gamma^{\mu}\left(i \partial_{\mu}-e \bar{A}_{\mu}\right) \psi+\frac{1}{4} \operatorname{tr} \widetilde{F}_{\mu \nu} \widetilde{F}^{\mu \nu}+\frac{1}{4} \operatorname{tr} \widetilde{M}_{\mu \nu} \widetilde{M}^{\mu \nu} \\
& -\frac{1}{4} \operatorname{tr} \widetilde{F}_{\mu \nu} \widetilde{M}^{\mu \nu}-\frac{1}{4} \operatorname{tr} \widetilde{M}_{\mu \nu} \widetilde{F}^{\mu \nu}-\frac{1}{4} \operatorname{tr} \widetilde{N}^{2}+\frac{N e^{2}}{2 g^{2}} \operatorname{tr} \bar{B}_{\mu} \bar{B}^{\mu},
\end{aligned}
$$

where we defined

$$
\begin{aligned}
& \widetilde{F}_{\mu \nu}=\partial_{\mu} \bar{A}_{\nu}-\partial_{\nu} \bar{A}_{\mu}+e\left[\bar{A}_{\mu}, \bar{A}_{\nu}\right], \\
& \widetilde{M}_{\mu \nu}=\partial_{\mu} \bar{B}_{\nu}-\partial_{\nu} \bar{B}_{\mu}+e\left[\bar{B}_{\mu}, \bar{B}_{\nu}\right],
\end{aligned}
$$

and the crossing noncommuting terms are given by

$$
\begin{aligned}
\widetilde{N}^{2} & =f_{d e}^{a}\left(\widetilde{F}_{\mu \nu}^{a}-\widetilde{M}_{\mu \nu}^{a}\right) t^{a}\left(\bar{A}^{\mu d} \bar{B}^{\nu e}+\bar{B}^{\mu d} \bar{A}^{\nu e}\right) t^{a} \\
& =f_{b c}^{a}\left(\bar{A}^{\mu b} \bar{B}^{\nu c}+\bar{B}^{\mu b} \bar{A}^{\nu c}\right) t^{a}\left(\widetilde{F}_{\mu \nu}^{a}-\widetilde{M}_{\mu \nu}^{a}\right) t^{a} \\
& =f_{b c}^{a}\left(\bar{A}^{\mu b} \bar{B}^{\nu c}+\bar{B}^{\mu b} \bar{A}^{\nu c}\right) t^{a} f_{d e}^{a}\left(\bar{A}_{\mu}^{d} \bar{B}_{\nu}^{e}+\bar{B}_{\mu}^{d} \bar{A}_{\nu}^{e}\right) t^{a} .
\end{aligned}
$$

Following similar paths as that of the Abelian cases, we redefine the fields as

$$
\begin{gathered}
\psi(x)=\exp \left[i \eta_{0}(x)+\gamma_{5} \phi_{0}(x)\right] \chi(x) \equiv U_{0} \chi(x), \\
\bar{\psi}(x)=\bar{\chi}(x) \exp \left[-i \eta_{0}(x)+\gamma_{5} \phi_{0}(x)\right] \equiv \bar{\chi}(x) U_{0}^{*}, \\
\overline{A_{\mu}^{0}}=\sqrt{\frac{N}{e}}\left(\epsilon_{\mu \nu} \partial^{\nu} \phi_{0}-\partial_{\mu} \eta_{0}\right) .
\end{gathered}
$$

In this way, we can decouple the U(1) sector of the theory: 


$$
\begin{aligned}
\overline{\mathcal{L}}= & \bar{\chi} \gamma^{\mu} \mathbb{D}^{\prime} \chi+\frac{1}{4} \operatorname{tr} \widetilde{F}_{\mu \nu} \widetilde{F}^{\mu \nu}+\frac{1}{4} \operatorname{tr} \widetilde{M}_{\mu \nu} \widetilde{M}^{\mu \nu}-\frac{1}{4} \operatorname{tr} \widetilde{F}_{\mu \nu} \widetilde{M}^{\mu \nu} \\
& -\frac{1}{4} \operatorname{tr} \widetilde{M}_{\mu \nu} \widetilde{F}^{\mu \nu}-\frac{1}{4} \operatorname{tr} \widetilde{N}^{2}+\frac{N e^{2}}{2 g^{2}} \operatorname{tr} \bar{B}_{\mu} \bar{B}^{\mu} \\
& +\frac{e^{2}}{2 \pi} \bar{A}_{\mu}^{0}\left(\delta^{\mu \nu}-\frac{\partial^{\mu} \partial^{\nu}}{\square}\right) \vec{A}_{\nu}^{0}+\text { noncomm. terms, }
\end{aligned}
$$

where $\mathbb{D}^{\prime}=i \not \partial-e \bar{A}^{\prime}, \bar{A}_{\mu}^{\prime}=\bar{A}_{\mu}^{a}, a=1, \ldots, N^{2}-1$, and the last term of the Lagrangian density comes from the Abelian part of the nontrivial Jacobian associated with transformations (4.9)-(4.11) [4].

Decomposing the Abelian part of gauge fields as

$$
e \bar{A}_{\mu}^{0}=\partial_{\mu} \eta_{A}+\epsilon_{\mu \nu} \partial^{\nu} \chi_{A}, \quad e \bar{B}_{\mu}^{0}=\partial_{\mu} \eta_{B}+\epsilon_{\mu \nu} \partial^{\nu} \chi_{B},
$$

we can write explicitly

$$
\mathcal{L}=\mathcal{L}_{\text {Abel }}+\mathcal{L}_{\text {non-Abel }},
$$

where (apart from harmonic excitations and source terms)

$$
\begin{aligned}
\mathcal{L}_{\text {Abel }}= & \frac{1}{2 e^{2}}\left[\left(\square \chi_{A}\right)^{2}+\left(\square \chi_{B}\right)^{2}\right]-\frac{1}{e^{2}} \square \chi_{A} \square \chi_{B} \\
& +\frac{N}{2 g^{2}}\left(\partial_{\mu} \chi_{B}\right)^{2}+\frac{1}{2 \pi}\left(\partial_{\mu} \chi_{A}\right)^{2},
\end{aligned}
$$

which is completely analogous to the vector model discussed above apart from the dependence on the rank of the nonAbelian group $\mathrm{SU}(N)$. Note that $\mathcal{L}_{\text {non-Abel }}$ retains all other terms present in Eq. (4.12). Doing the transformations

$$
\chi_{A} \rightarrow a_{N} \bar{\chi}_{A}, \quad \chi_{B} \rightarrow \bar{\chi}_{B}, \quad a_{N}=\sqrt{\frac{N \pi}{g^{2}}},
$$

and a rotation analogous to Eq. (2.10) with $\tan \phi=1 / a_{N}$, we find

$$
\mathcal{L}_{\text {Abel }}=\frac{a_{N}^{2}+1}{2 e^{2}} \chi_{A}^{\prime}\left(\square^{2}-m^{2} \square\right) \chi_{A}^{\prime}-\frac{N}{2 g^{2}} \chi_{B}^{\prime} \square \chi_{B}^{\prime},
$$

where the dynamically generated mass in this case is given by

$$
m^{2}=\frac{N e^{2}}{N \pi+g^{2}}
$$

From this result, one can see that in the weak coupling limit, $g \rightarrow 0$, we reobtain the Schwinger mass (2.15). This is, in some sense, expected since we have only calculated the Abelian contribution to the dynamically generated mass. In the strong coupling limit $(g \rightarrow \infty)$, we also find a reduction in the mass analogous to the Abelian vector case, Eq. (2.16), and the duality between weak gauge coupling and strong quartic self-coupling. In addition, in this non-Abelian case we find a dependence on the group rank parameter $N$. For large $N$, the contribution of the quartic self-interaction term disappears and we recover again the Schwinger mass, which shows that this non-Abelian model in the strong coupling limit resembles, in some sense, the large $N$ limit of $\mathrm{QCD}_{2}$ [19]. Note also that the large $N$ and the weak $g$ limits are equivalent.

\section{OPERATOR SOLUTION OF THE VECTOR MODEL}

Let us discuss the vectorial case (the left and right couplings are equal) and avoid the anomaly keeping the gauge invariance in the bosonization of the model. The Lagrangian density will be, in this case,

$$
L=-\frac{1}{4} F^{\mu \nu} F_{\mu \nu}+\imath \bar{\psi} \mathbb{D} \psi-\frac{g^{2}}{2} \bar{\psi} \gamma^{\mu} \psi \bar{\psi} \gamma_{\mu} \psi .
$$

Analogously to the solution of the Thirring model from the free fermionic model, we will use the interaction picture [21] using as free model the Schwinger model [2].

The solution of the Schwinger model in the Lorentz gauge is

$$
\begin{gathered}
\psi_{i}=\sqrt{\mu / 2 \pi} \times: \exp \left(\imath \sqrt{\pi} \gamma_{i, i}^{5}\left(-\alpha_{1}-\alpha_{2}+\alpha_{3}\right)\right. \\
\left.-\imath \sqrt{\pi} \int_{x}^{\infty} \dot{\alpha}_{3} d y^{1}\right) \\
A^{\mu}=-\frac{1}{m} \epsilon^{\mu \nu} \partial \nu\left(\alpha_{1}+\alpha_{2}\right) .
\end{gathered}
$$

Here, $\alpha_{1}$ is a canonical scalar field with mass $m=e / \sqrt{\pi}, \alpha_{3}$ is a canonical massless field, and $\alpha_{2}$ the same, but with negative metric, while $\mu$ is an infrared regulator. The Maxwell equation in the physical subspace is satisfied with the current

$$
J^{\mu}=\frac{1}{\sqrt{\pi}} \epsilon^{\mu \nu} \partial \nu\left(\alpha_{1}+\alpha_{2}-\alpha_{3}\right) .
$$

These fields define the operator solution to the above Lagrangian in the interaction picture. To boost them to the Heisenberg picture while keeping the Lorentz invariance, it is necessary first to verify the validity of the Schwinger condition. This requires that the energy-momentum tensor satisfies the commutation relation $\left[\Theta_{00}(x), \Theta_{00}(y)\right]$ $={ }_{l}\left[\Theta_{01}(x)+\Theta_{01}(y)\right] \partial_{x} \delta(x-y)[21]$. The Schwinger model Hamiltonian

$$
\begin{aligned}
\Theta_{0,0}^{\text {free }}= & (1 / 2)\left[\left(\partial_{t} \alpha_{1}\right)^{2}-\left(\partial_{t} \alpha_{2}\right)^{2}+\left(\partial_{t} \alpha_{3}\right)^{2}+\left(\partial_{x} \alpha_{1}\right)^{2}\right. \\
& \left.-\left(\partial_{x} \alpha_{2}\right)^{2}+\left(\partial_{x} \alpha_{3}\right)^{2}+\left(e^{2} / 2 \pi\right)\left(\alpha_{1}\right)^{2}\right]
\end{aligned}
$$

is easily seen to verify this condition. The only nonvanishing term in the interaction energy-momenta tensor is

$$
\Theta_{00}^{\mathrm{int}}=\frac{g^{2}}{2}\left[\left(J^{0}\right)^{2}-b\left(J^{1}\right)^{2}\right]
$$

where the factor $b$ would not appear if we naively computed current-current product in the interaction picture. Requiring the validity of the Schwinger condition, we fix the value $b=1 /\left(g^{2} / \pi+1\right)$. This same phenomenon of introducing an apparently noncovariant definition of the interaction appears 
in the analogous treatment to the Thirring model (without the gauge field) and the value of $b$ turns out to be the same [21]. Now, we take the free Hamiltonian expressed in terms of the fields $\alpha_{i}$ and their conjugate momenta $\Pi_{i}$ (note that $\Pi_{2}=-\dot{\alpha}_{2}$ ), add the interaction term resulting in a Hamiltonian that, in spite of quadratic, has crossed terms among all fields and all momenta. In the Heisenberg picture the new definition of the momenta can be obtained inverting the canonical equations $\left(\partial_{t} \alpha_{j}=\partial_{\Pi_{j}} H\right)$. They are

$$
\begin{gathered}
\Pi_{1}=\left(1+\frac{g^{2}}{\pi}\right) \dot{\alpha}_{1}+\frac{g^{2}}{\pi}\left(\dot{\alpha}_{2}-\dot{\alpha}_{3}\right), \\
\Pi_{2}=-\left(1-\frac{g^{2}}{\pi}\right) \dot{\alpha}_{2}+\frac{g^{2}}{\pi}\left(\dot{\alpha}_{1}-\dot{\alpha}_{3}\right), \\
\Pi_{3}=\left(1+\frac{g^{2}}{\pi}\right) \dot{\alpha}_{3}-\frac{g^{2}}{\pi}\left(\dot{\alpha}_{2}+\dot{\alpha}_{1}\right) .
\end{gathered}
$$

Now, the original variables $\alpha_{i}$ are not free fields. Let us then perform a generic linear transformation to new variables. Requiring canonical commutation relations for these new fields, we chose the transformations as:

$$
\begin{gathered}
\alpha_{1}=\frac{1}{\sqrt{1+g^{2} / \pi}} \phi_{1}, \\
\alpha_{2}=\sqrt{1+g^{2} / \pi} \phi_{2}+\frac{g^{2}}{\pi \sqrt{1+g^{2} / \pi}} \phi_{1}, \\
\alpha_{3}=\frac{1}{\sqrt{1+g^{2} / \pi}} \phi_{3}+\frac{g^{2}}{\pi \sqrt{1+g^{2} / \pi}}\left(\phi_{1}+\phi_{2}\right) .
\end{gathered}
$$

Substituting these definitions in the Hamiltonian results in:

$$
\begin{aligned}
H= & \frac{1}{2}\left[\left(\partial_{t} \phi_{1}\right)^{2}+\left(\partial_{x} \phi_{1}\right)^{2}+m_{a}{ }^{2} \phi_{1}{ }^{2}-\left(\partial_{t} \phi_{2}\right)^{2}-\left(\partial_{x} \phi_{2}\right)^{2}\right. \\
& \left.+\left(\partial_{t} \phi_{3}\right)^{2}+\left(\partial_{x} \phi_{3}\right)^{2}\right] .
\end{aligned}
$$

We see that $\phi_{1}$ is a canonical field with mass equal to $m(g)=m / \sqrt{1+g^{2} / \pi}, \phi_{2}$ is a negative metric, and $\phi_{3}$ a positive metric canonical massless field. This mass coincides with the one in Eq. (2.15) when $\lambda=1$. The solution of the model is obtained from the free one, upon the substitution of the old phase space variables by the new ones. The result is

$$
\begin{gathered}
\psi_{j}=N: \exp \left(\frac{-\imath \pi}{\sqrt{\pi+g^{2}}} \gamma_{j, j}^{5}\left(\phi_{1}+\phi_{2}-\phi_{3}\right)\right. \\
\left.-\imath \sqrt{\pi+g^{2}} \int_{x}^{\infty} \dot{\phi}_{3} d \xi^{1}\right), \\
A^{\mu}=-\frac{1}{m(g)} \epsilon^{\mu \nu} \partial \nu\left(\phi_{1}+\phi_{2}\right) .
\end{gathered}
$$

From them any correlation function may be easily computed.
The current cannot be obtained simply taking the free one and substituting the old fields and momenta by the new ones. As in the Thirring model case, we have to redefine the $J_{1}$ component with a $1 / \sqrt{1+g^{2} / \pi}$ factor. Only after that the current becomes vectorial and results in

$$
J^{\mu}=\frac{1}{\sqrt{\pi+g^{2}}} \epsilon^{\mu \nu} \partial_{\nu}\left(\phi_{1}+\phi_{2}-\phi_{3}\right) .
$$

This current can be also obtained computing the short distance gauge-invariant operator product of the fermion fields and normalizing them to have a vectorial field.

We can verify that the Dirac equation becomes satisfied, IID $\psi=g^{2} \boldsymbol{J}$. The vector field satisfies the equation

$$
\partial_{\mu} F^{\mu \nu}-e J^{\nu}=e L^{\nu} .
$$

As in the Schwinger model, the $L^{\mu}$ current is a null metric field. The Maxwell equation is obeyed in the physical subspace where the expectation values of this current are zero:

$$
L^{\mu}=\frac{1}{\sqrt{\pi+g^{2}}} \epsilon^{\mu \nu} \partial_{\nu}\left(\phi_{3}-\phi_{2}\right) .
$$

In order to obtain the spurious operators, one can consider the operators that would result from a gauge transformation:

$$
\begin{gathered}
\psi_{j}=N: \exp \left(\frac{-\imath}{\sqrt{\pi+g^{2}}} \gamma_{j, j}^{5}\left(\phi_{1}+\phi_{2}-\phi_{3}\right)\right. \\
\left.-\imath \sqrt{\pi+g^{2}} \int_{x}^{\infty}\left(\dot{\phi}_{3}-\dot{\phi}_{2}\right) d y^{1}\right), \\
A^{\mu}=-\frac{1}{m(g)} \epsilon^{\mu \nu} \partial \nu \phi_{1} .
\end{gathered}
$$

From Eqs. (5.19), we see that the fermion operators violate the cluster property. As the field $\phi_{1}$ belongs to the operator algebra of the model [17], we can factorize its contribution out of the fermionic fields. The resulting operators commute with the null metric current $L_{\mu}$ and have constant correlation functions. They define the two spurious operators after the introduction of interaction:

$$
\begin{aligned}
\sigma_{j}= & N: \exp \left(\frac{\imath}{\sqrt{\pi+g^{2}}} \gamma_{j, j}^{5}\left(-\phi_{2}+\phi_{3}\right)\right. \\
& \left.-\imath \sqrt{\pi+g^{2}} \int_{x}^{\infty}\left(\dot{\phi}_{3}-\dot{\phi}_{2}\right) d y^{1}\right) .
\end{aligned}
$$

These operators expose the origin of the violation of the cluster property. In order to restore it, one should define the $\theta$ vacua. As in the Schwinger model, this would lead to a set of degenerate vacua. The spurious operators $\sigma_{1}$ and $\sigma_{2}$ differ from the ones in the Schwinger model as they are not chiral, however. The effect of the interaction appears in their dependence at the same time of the right and left light-cone variables. The dependence of the solution on the light-cone variables can be clarified with one question: The operators $\sigma_{j}$ are not chiral, but are functions of them that depend only on left 
or right variables? For the answer, note that the general spurious operator will be an arbitrary integer power of these. Taking, for instance, $\sigma_{1}^{n_{1}} \sigma_{2}^{-n_{2}}$ and requiring no dependence on $x-t$, results that $n_{1} / n_{2}$ shall equal $g /(g+2)$. When $g=0$, this means that $\sigma_{2}$ is chiral. In the generic case this equation cannot be satisfied, as $g$ may be an irrational number. Nevertheless, as the rationals are dense in the reals, there will ever be a value of $g$ arbitrarily close to any prescribed value that makes it possible to construct chiral spurious operators. On the other hand, we could deal only with the operators present in the algebra of fields of the model and avoid the introduction of the gauge transformation leading to the pair of spurious operators. In this case, the unique spurious operator will be $\sigma_{1}^{\dagger} \sigma_{2}$ where the effect of interaction will show up in its dimension being changed.

Let us now construct the generators of gauge transformations of the model. Following [22] we take the generators of gauge transformation of the Schwinger model and boost them to the Heisenberg picture. In the interaction picture one has

$$
\begin{aligned}
T(\lambda)= & \exp \left(\frac { l } { \sqrt { \pi } } \int d y ^ { 1 } \left[\left(\alpha_{3}+\alpha_{2}\right) \partial_{1} \lambda(y)\right.\right. \\
& \left.\left.-\left({\widetilde{\alpha_{3}}}+{\widetilde{\alpha_{2}}}\right) \partial_{0} \lambda\right]\right),
\end{aligned}
$$

where $\widetilde{\alpha}_{3}(x)=-\int_{x}^{\infty} d y^{1} \Pi_{3}(\chi)$ and $\widetilde{\alpha}_{2}(x)=\int_{x}^{\infty} d y^{1} \Pi_{2}(\chi)$. Performing the change to the Heisenberg picture and expressing in terms of the new variables, we obtain the generators of gauge transformations in the interacting model:

$$
\begin{aligned}
T(\lambda)= & \exp \left(\frac { l } { \sqrt { \pi + g ^ { 2 } } } \int d y ^ { 1 } \left[\left(\phi_{3}+\phi_{2}\right) \partial_{1} \lambda(y)\right.\right. \\
& \left.\left.-\left(\widetilde{\phi}_{3}+\widetilde{\phi}_{2}\right) \partial_{0} \lambda\right]\right)
\end{aligned}
$$

Commuting with the fields, we can ascertain that these are the correct generators of the gauge transformations:

$$
\begin{gathered}
T \psi(x) T^{-1}=e^{\imath \lambda(x)} \psi(x), \\
{\left[T, A_{\mu}(x)\right]=-\frac{1}{e} \partial_{\mu} \lambda(x) .}
\end{gathered}
$$

Following [12], we define $\lambda_{-1 / 2}\left(y^{0}, y^{1}\right)=-\pi \Theta\left(y^{0}-y^{1}\right.$ $\left.-x^{-}\right)$and $\lambda_{1 / 2}\left(y^{0}, y^{1}\right)=-\pi \Theta\left(y^{0}+y^{1}-x^{+}\right)$. Taking $\lambda_{1}=\left(1+g^{2} / 2 \pi\right) \lambda_{1 / 2}+\left(g^{2} / 2 \pi\right) \lambda_{-1 / 2}$, we see that this generator reduces to the spurious operator $\sigma_{1}$, while $\lambda_{2}=\left(1+g^{2} / 2 \pi\right) \lambda_{-1 / 2}+\left(g^{2} / 2 \pi\right) \lambda_{1 / 2}$ leads to the $\sigma_{2}$ operator. As in the Schwinger model, the screening is due to the disappearance, after the switching to the $\theta$ vacua, of the physical states carrying quantum numbers associated to the generators of $\mathrm{U}(1)$ and chiral $\mathrm{U}(1)$ gauge transformations. The difference is that the basic generators are not chiral any more. In any case, looking at the true spurious operators obtained with the fields that define the gauge-invariant algebra of the operators of the model, $\sigma_{1}^{*} \sigma_{2}$, we see that they are, as in the Schwinger model, functions of the scalar op- erator $\phi_{2}-\phi_{1}$ and not of their chiral components. Let us note that, although the vectorial field expression could be obtained directly from the Schwinger model vector field through a simple redefinition of the electric coupling constant, as shown in the second section, the fermionic field cannot be obtained so simply with the same transformation. Further, the gauge-invariant interacting two-point fermionic functions cannot be obtained from the ones in the Schwinger model through such a transformation. The effect of the interaction after quantization is to define a new class of models.

The value of the chiral anomaly can now be computed taking $J_{5}^{\mu}=\epsilon^{\mu \nu} J_{\nu}$. We have

$$
\partial_{\mu} J_{5}^{\mu}=\frac{-m(g)}{2 \sqrt{\pi+g^{2}}} \epsilon_{\mu \nu} F^{\mu \nu}=\frac{-e}{2\left(\pi+g^{2}\right)} \epsilon_{\mu \nu} F^{\mu \nu} .
$$

The spatial integral of $J_{5}^{0}$ gives not properly the chiral transformation generator $Q_{5}$. To have $\left[Q_{5}, \psi\right]=\imath \gamma_{5} \psi$, the current should be multiplied by $1+g^{2} / \pi$, reflecting directly in the value of the anomaly.

\section{OPERATOR SOLUTION OF THE CHIRAL MODEL}

Let us turn now to operator solution of the Abelian chiral case. The fermionic Lagrangian is

$$
\begin{aligned}
L= & -\frac{1}{4} F^{\mu \nu} F_{\mu \nu}+\imath \bar{\psi} \mathbb{D}(+) \psi-\frac{G \pi}{2 a} \bar{\psi} \gamma^{\mu}\left(1+\gamma_{5}\right) \\
& \times \psi \bar{\psi} \gamma_{\mu}\left(1+\gamma_{5}\right) \psi,
\end{aligned}
$$

where $D_{(+)}=\gamma^{\mu} \partial_{\mu}+\imath \gamma^{\mu} A_{\mu}\left(1+\gamma_{5}\right)$ and it is convenient to define the coupling through $G=g^{2} a / \pi$. In this case, it is more straightforward to bosonize directly the Lagrangian obtaining the equivalent model

$$
\begin{aligned}
L= & -\frac{1}{4} F^{\mu \nu} F_{\mu \nu}+\frac{1}{2} \partial_{\mu} \phi \partial^{\mu} \phi+\frac{e}{\sqrt{\pi}}\left(\partial_{\mu}+\widetilde{\partial}_{\mu}\right) \phi A^{\mu} \\
& +\frac{a e^{2}}{2 \pi} A_{\mu} A^{\mu}-\frac{g^{2}}{2} J_{\mu} J^{\mu} .
\end{aligned}
$$

In the operator approach the bosonized expression of the current depends largely on the regularization used on its computation. We choose the current that would appear in the Maxwell equation in the $g=0$ model. Namely, we take $\bar{\psi} \gamma_{\mu}\left(1+\gamma_{5}\right) \psi \approx J_{\mu}=(-1 / \sqrt{\pi})\left[\left(\widetilde{\partial}_{\mu}+\partial_{\mu}\right) \phi+(e a / \sqrt{\pi}) A_{\mu}\right]$. This regularization differs from the one used in the computation of the $J_{\mu} A^{\mu}$ term as the contribution of the very vector field to it is taken with different weight. We could have worked with the same regularization without changing the main course of arguments, but the expressions involved would be unnecessarily cumbersome to read. Substituting $A_{\mu}=\widetilde{\partial}_{\mu} \chi+\partial_{\mu} \lambda$ in the Lagrangian and changing variables to

$$
\begin{gathered}
\phi=\phi_{3}-\frac{e}{\sqrt{\pi}}(1-G) \lambda_{1}+\frac{e(1-G) a}{\sqrt{\pi}(a-1+G)} \chi, \\
\lambda=\lambda_{1}-\frac{1-G}{a-1+G} \chi
\end{gathered}
$$


results in

$$
\begin{aligned}
L= & \frac{1}{2} \square \chi \square \chi-\frac{m_{g}{ }^{2}}{2} \partial_{\mu} \chi \partial^{\mu} \chi+\frac{1}{2} \partial_{\mu} \phi_{3} \partial^{\mu} \phi_{3} \\
& +\frac{e^{2}(1-G)(a-1+G)}{2 \pi} \partial_{\mu} \lambda_{1} \partial^{\mu} \lambda_{1},
\end{aligned}
$$

where the mass $m_{g}=e \sqrt{1-G} a / \sqrt{a-1+G} \sqrt{\pi}$ coincides with the one computed in Eq. (3.9) upon properly choosing the parameters $\alpha$ and $\beta$. The solution of the higher derivative field $\chi$ is well known [8], leading to $\chi=\left(\phi_{2}-\phi_{1}\right) / m_{g}$ where the field $\phi_{1}$ has mass $m_{g}$ and positive metric, while the massless field $\phi_{2}$ is quantized with negative metric. The solution of the model is obtained expressing the free fermionic field in terms of $\phi$ and its momentum and substituting the latter by $\partial_{\dot{\phi}} L$, so we find

$$
\begin{aligned}
A_{\mu}= & \frac{1}{m_{g}}\left(\tilde{\partial}_{\mu}\left(\phi_{2}-\phi_{1}\right)-\frac{(1-G)}{(a-1+G)} \partial_{\mu}\left(\phi_{2}-\phi_{1}\right)\right) \\
& +\partial_{\mu} \lambda_{1}, \\
& \psi_{2}=\exp \left[-\imath \sqrt{\pi}\left(\phi_{3}+\int_{x}^{\infty} d y^{1} \phi_{3}\right)\right], \\
\psi_{1}= & \exp \left[\imath \sqrt { \pi } \left(\phi_{3}+2 \sqrt{\frac{1-G}{\sqrt{a-1+G}}}\left(\phi_{2}-\phi_{1}\right)\right.\right. \\
& \left.\left.-\frac{2 e(1-G)}{\pi} \lambda_{1}-\int_{x}^{\infty} d y^{1} \phi_{3}\right)\right] .
\end{aligned}
$$

The Dirac equation will read $\imath \partial_{+} \psi_{2}=0$ and $\left(\imath \partial_{-}-2 A_{-}-2 g^{2} J_{-}\right) \psi_{1}=0$. The Maxwell equation will be $\partial_{\mu} F^{\mu \nu}=e(1-G) J^{\nu}+e(1-G) L^{\nu}$, where the current is

$$
\begin{aligned}
J_{\mu}= & \frac{-1}{\sqrt{\pi}}\left(\left(\partial_{\mu}+\widetilde{\partial}_{\mu}\right) \phi_{3}+\frac{a}{\sqrt{a-1+G} \sqrt{1-G}} \widetilde{\partial}_{\mu}\left(\phi_{2}-\phi_{1}\right)\right. \\
& \left.+\frac{e}{\sqrt{\pi}}\left[(a-1+G) \partial_{\mu} \lambda_{1}-(1-G) \widetilde{\partial}_{\mu} \lambda_{1}\right]\right)
\end{aligned}
$$

and the null metric current given by

$$
\begin{aligned}
L_{\mu}= & \frac{1}{\sqrt{\pi}}\left(\left(\partial_{\mu}+\tilde{\partial}_{\mu}\right) \phi_{3}+\frac{a}{\sqrt{a-1+G} \sqrt{1-G}} \tilde{\partial}_{\mu} \phi_{2}\right. \\
& \left.+\frac{e}{\sqrt{\pi}}\left[(a-1+G) \partial_{\mu} \lambda_{1}-(1-G) \tilde{\partial}_{\mu} \lambda_{1}\right]\right)
\end{aligned}
$$

determines the physical subspace out of the complete Hilbert space. The physical fields turn out to be $\phi_{1}$ and $h=\phi_{3}-[e(1-G) / \sqrt{\pi}] \lambda_{1}+\sqrt{(1-G) /(a-1+G)} \phi_{2}$. It is interesting to note that the introduction of the current-current interaction in this case can be completely accommodated in a redefinition of the parameters of the original $(g=0)$ Lagrangian. Indeed, making $e \rightarrow e(1-G)$ and $a \rightarrow a /(1-G)$ in the chiral Schwinger model solution leads us directly to the interacting $(g \neq 0)$ model solution. This can be understood as a consequence of the fact that the interaction term $J_{\mu} J^{\mu}$ would be naively zero if one used the chiral current expressed only in terms of the fermion fields, as this product would be identically zero. What makes this interaction different from zero is exactly the ambiguity in the definition of the currents present in the chiral Schwinger model. This phenomenon has its correspondence in the functional formalism in the fact that the introduction of an identity, $1=\exp \left(\left\langle J_{\mu} J^{\mu}\right\rangle\right)$, in the integrand together with the ambiguities in the Jacobian leads to the redefinition of the parameters of the model.

It is important to note that in the chiral case we cannot define an spurious operator in terms of the algebra of operators that are observables of the theory. There is no $\theta$ vacuum in this case, as happens in the usual chiral model, without quartic interaction.

\section{CONCLUSION}

We have analyzed the effect of current-current coupling in some models of fermion fields interacting with gauge fields. In the three cases (vector, chiral, and non-Abelian models), we find that the dynamically generated mass is modified by the quartic self-interaction coupling. The three models also show a kind of duality between the gauge and quartic couplings. The gauge field correlation functions in the weak gauge $(e \rightarrow 0)$ coupling are the same as the ones in the strong $(g \rightarrow \infty)$ quartic coupling. Whether this relation between gauge and quartic couplings also appears in higher dimensions deserves to be further investigated, but the question of renormalizability in this case will be a drastic one.

Furthermore, in the non-Abelian case, the dynamically generated mass is also dependent on the gauge group rank $N$ and displays additional interesting limits. For the large $N$ limit, the mass reduces to the usual Schwinger one, resembling the exact solvability of large $N \mathrm{QCD}_{2}$.

Specifically, in the case of the vector model the introduction of the $g$ coupling changes the mass acquired through the Schwinger mechanism. Using functional methods, we computed the vector field correlation functions. Using operator methods, the fermions and vector fields are obtained confirming the results in the functional treatment. The Maxwell equations are obeyed in the physical subspace, where the null metric current that depends explicitly on $g$ has vanishing correlation functions. The spurious operators were then constructed, leading to $\theta$ vacua. They are shown to be related to the gauge generators of the model whereupon the same screening mechanism is observed. The chiral anomaly is computed and shows the dependence on $g$. These results complement the approach in [23] where the order-disorder variables of some two-dimensional models are studied. The decoupling of the Abelian gauge field in non-Abelian models with current-current interaction is obtained in analogous fashion to the $g=0$ case.

In the chiral case, in contrast with the vectorial Schwinger model, the introduction of the coupling can be accommodated in a redefinition of the parameters of the chiral Schwinger model. Although the $g$ interaction Hamiltonian would be identically zero if there were no gauge interaction, it survives quantization as shown in the functional and operatorial formalism. In the first case, it occurs through the arbitrariness in the definition of the Jacobian in the decou- 
pling transformation and in the operatorial case, it occurs through the arbitrariness in the definition of the currents. This phenomenon should be taken as an alert to the care one should have when dealing with interacting fields. For instance, if instead of considering the chiral model, one deals with the generalized one, with arbitrary left and right couplings, the current-current term would not be identically zero. By continuously changing the couplings to approach the model to the chiral model, one should expect the quartic interaction to disappear only if there is no gauge interaction present, or by carefully dealing with the Jackiw-Rajaraman ambiguity term.

\section{ACKNOWLEDGMENTS}

One of the authors (C.P.N.) would like to acknowledge the financial support of UNESP. The authors were partially supported by CNPq, Brazilian agency.
[1] J. Schwinger, Phys. Rev. 128, 2425 (1962).

[2] J. H. Lowenstein and J. Swieca, Ann. Phys. (N.Y.) 68, 112 (1971).

[3] K. Fujikawa, Phys. Rev. Lett. 42, 1195 (1979); Phys. Rev. D 21, 2848 (1980).

[4] R. E. Gamboa-Saravi, F. A. Schaposnik, and J. E. Solomin, Nucl. Phys. B185, 239 (1981).

[5] R. Jackiw and R. Rajaraman, Phys. Rev. Lett. 54, 1219 (1985).

[6] R. Rajaraman, Phys. Lett. 154B, 305 (1985); A. Niemi and G. Semenoff, Phys. Lett. B 175, 439 (1986); C. A. Aragão de Carvalho, K. D. Rothe, C. A. Linhares, and H. J. Rothe, ibid. 194, 539 (1987); K. Shizuya, ibid. 213, 298 (1988); H. O. Girotti and K. D. Rothe, Int. J. Mod. Phys. A 6, 3041 (1989); L. V. Belvedere and K. D. Rothe, Mod. Phys. Lett. A 10, 207 (1995).

[7] M. Chanowitz, Phys. Lett. B 171, 280 (1986); see also A. de Souza Dutra, Int. J. Mod. Phys. A 9, 2229 (1994).

[8] D. Boyanovsky, I. Schmidt, and M. F. L. Goltterman, Ann. Phys. (N.Y.) 185, 111 (1988).

[9] W. Thirring, Ann. Phys. (N.Y.) 3, 91 (1958).

[10] B. Klaiber, in Lectures in Theoretical Physics, Boulder, 1967, edited by A. O. Barut (Gordon and Breach, New York, 1968).

[11] D. Gross and A. Neveu, Phys. Rev. D 10, 3235 (1974).
[12] K. D. Rothe, E. Abdalla, and M. Abdalla, Theoretical Methods in Two Dimensional Quantum Field Theory (World Scientific, Singapore, 1992).

[13] L. V. Belvedere, Nucl. Phys. B276, 197 (1986).

[14] See, for instance, M. B. Green, J. H. Schwarz, and E. Witten, Superstring Theory (Cambridge University Press, Cambridge, England, 1987).

[15] N. Seiberg, Nucl. Phys. B426, 19 (1994); N. Seiberg and E. Witten, ibid. B431, 484 (1994).

[16] A. Dettki, I. Sachs, and A. Wipf, Report No. ETH-TH/93-14, hep-th/9308067, 1993 (unpublished).

[17] L. V. Belvedere, C. P. Natividade, C. G. Carvalhaes, and H. Boschi Filho, "Algebraic Isomorphism in Two-Dimensional Gauge Theories," report (in preparation).

[18] C. P. Natividade, A. de Souza Dutra, and H. Boschi-Filho, Z. Phys. C 67, 687 (1995).

[19] E. Witten, Nucl. Phys. B145, 110 (1978).

[20] K. Furuya, R. E. Gamboa-Saravi, and F. A. Schaposnik, Nucl. Phys. B208, 159 (1982).

[21] M. B. Halpern, Phys. Rev. D 12, 1684 (1975); 13, 337 (1976).

[22] K. D. Rothe and J. A. Swieca, Phys. Rev. D 15, 541 (1977).

[23] E. C. Marino, Ph.D. thesis, Pontifícia Universidade Católica des Rio de Janeiro, 1980. 